\title{
An Autopsy Case of Dementia with Lewy Bodies with Vocal Cord Abductor Paralysis
}

\author{
Shuta Toru ${ }^{b}$ Toshiki Uchihara $^{a}$ Makoto Hara ${ }^{a}$ Sunao Mae ${ }^{c}$ Michio Toru $^{d}$ \\ Katsuiku Hirokawa ${ }^{e}$ Takashi Endo $^{f}$ Emiko Sugawara $^{f}$ Masanobu Kitagawa $^{f}$ \\ Takayoshi Kobayashi ${ }^{\text {b }}$ \\ a Laboratory of Structural Neuropathology, Tokyo Metropolitan Institute of Medical Science, and Departments of \\ ${ }^{b}$ Neurology, ' Medicine, dPsychiatry and e Pathology, Nitobe Memorial Nakano General Hospital, and ${ }^{\mathrm{f} D e p a r t m e n t}$ of \\ Pathology, Tokyo Medical and Dental University, Tokyo, Japan
}

Dear Sir,

Vocal cord abductor paralysis (VCAP), frequent in the later stage of multiple systemic atrophy (MSA) [1-5], is rare in Parkinson's disease (PD) $[5,8,9]$ and exceptional in dementia with Lewy bodies (DLB). Here we report the first autopsy-verified case of DLB with VCAP.

The patient felt anxiety at the age of 71, and consulted the psychiatrist of our hospital; mild dementia was diagnosed and minor tranquilizer was prescribed. At the age of 75, he experienced difficulty in playing tennis. Gradually, his walking slowed down. At the age of 76, he had visual hallucination. A neurologist of our hospital noted akinesia, mask-like face and small steppage gait and apparent dementia based on mini-mental state examination (MMSE $21 / 30)$. He was diagnosed as having probable DLB based on the clinical criteria [10]. L-DOPA was administered, but his symptoms progressed gradually. He had a percutaneous endoscopic gastrostomy because of aspiration pneumonia at the age of 81 . He became bedridden at the age of 83 . At the age of 87 , he exhibited inspiratory stridor, and endoscopic examination of the larynx disclosed bilateral VCAP. The cervi- cal - chest XP and CT revealed no lesion affecting the recurrent and/or vagus nerves. The family's consent to perform tracheotomy was not obtained. At the age of 89 , he was admitted to our hospital with pyrexia. On admission, neurological examination revealed new findings including right-sided dominant rigidity and Myerson's sign. Resting tremor was absent. He showed severe dementia (MMSE 0/30). Moderate-tosevere atrophy of fronto-temporal cortex including hippocampus and ischemic change were revealed on his brain MRI (fig. 1a). But brainstem-cerebellar atrophy, hot cross ban sign or abnormal intensity of striatum were absent. On hospital day 34, he died with uncontrolled infection.

Brain weight was 1,185 g. Macroscopically, the medial temporal lobe was atrophic, and moderate-to-severe depigmentation was apparent in the substantia nigra and locus ceruleus. Histologically, the brain stem (substantia nigra, locus ceruleus, dorsal motor nucleus of the vagus, raphe nuclei) was moderately to severely involved with Lewy pathology (fig. 1b). Although this pathological change extended to the ambiguus nucleus, the neuronal loss and gliosis of the ambiguus nucleus was at most slight (fig. 1c, d). Lewy pathology were also seen in the limbic system (hippocampus, amygdala, cingulate gyrus) moderately (fig. 1e), in the neocortex (superior temporal, insula, parietal, occipital gyri) mildly, and in the spinal cord (cervical to sacral segment) moderately. These were consistent with DLB of the neocortical type in pathological criteria [10]. Alzheimer pathology of Braak NFT stage II and amyloid stage B were also seen. There were no findings associated with old age hippocampal sclerosis. Intrinsic laryngeal muscles showed mild neurogenic change including atrophic fibers, angulated fibers, and small group atrophy (fig. 1f).

Anatomical tracing based on autopsy samples of MSA patients from ambiguus nucleus, myelinated fibers in the motor divisions of the laryngeal branch of the recurrent laryngeal nerve [6], neuromuscular junctions of the posterior cricoarytenoid muscle [7] and intrinsic laryngeal muscles indicate neurogenic paralysis of intrinsic laryngeal muscles as the underlying mechanism for VCAP in MSA. In contrast with frequent VCAP in MSA, VCAP is very rare in clinically diagnosed $\mathrm{PD}$ patients $[5,8,9]$,

\section{KARGER 125}

(c) 2015 S. Karger AG, Base

$0014-3022 / 15 / 0744-0186 \$ 39.50 / 0$
Dr. Shuta Toru

Department of Neurology, Nitobe Memorial Nakano General Hospital 4-59-16, Chuo, Nakano-ku, Tokyo 164-8607 (Japan)

E-Mail shuta-toru@nakanosogo.or.jp 

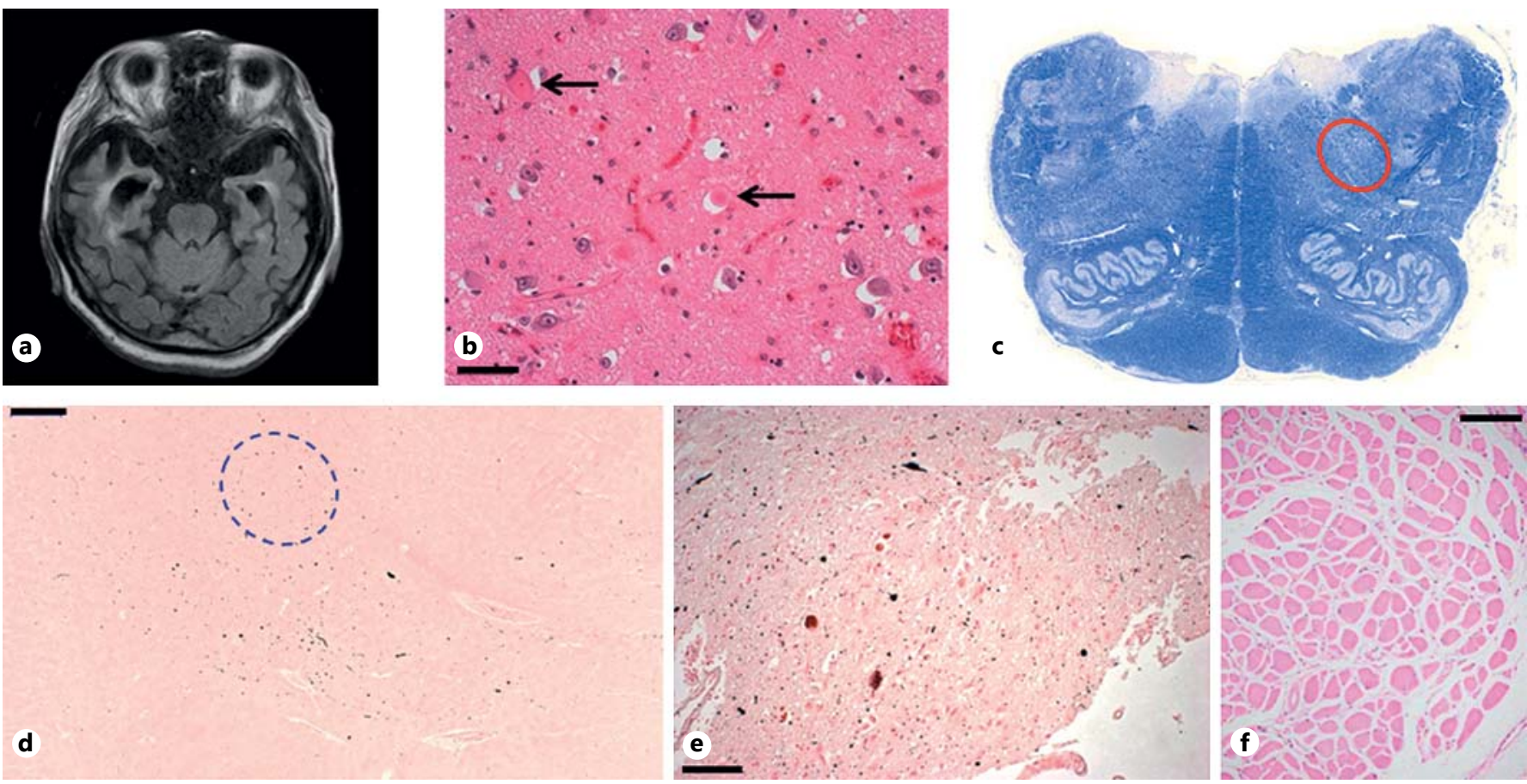

Fig. 1. a Brain MRI (FLAIR) showed medial temporal atrophy. b The arrows indicate Lewy bodies in the amygdala (HE). Scale bar $=50 \mu \mathrm{m}$. c Medulla oblongata of KB stain. Neuronal loss and gliosis were slight in nucleus ambiguus (red circle). $\mathbf{d} \alpha$-Synuclein positively stained structures in the intermediate reticular zone of

ventrolateral medulla. The dotted circle indicates nucleus ambiguus. Scale bar $=500 \mu \mathrm{m}$. e $\alpha$-Synuclein positively stained structures in locus ceruleus high-power field. Scale bar $=100 \mu \mathrm{m}$. $\mathbf{f}$ The posterior cricoarytenoid muscles showed only mild neurogenic findings (HE). Scale bar $=200 \mu \mathrm{m}$.

and has not yet been reported in clinically diagnosed DLB. Among them, autopsy-verified PD with VCAP was limited to a single report [5]. Because no remarkable changes were detected in the intrinsic laryngeal muscles of these PD patients with VCAP, sustained tonic state of intrinsic laryngeal muscles was considered to be responsible for VCAP in PD. In this DLB patient as well, there was only mild neurogenic change in intrinsic laryngeal muscles and neuronal loss was not evident in ambiguus nucleus, which alone cannot explain VCAP. We speculated that the same mechanism induced VCAP in PD and DLB.

This is the first autopsy report of DLB with VCAP. VCAP is a life-threatening complication, which may cause sudden death in patients. Therefore, even if VCAP is exceptionally rare in DLB, it is the condition of a patient that we should be careful about.

\section{Disclosure Statement}

The authors report no conflicts of interest. No funding was related to this work.

\section{References}

1 Israel RH, Marino JM: Upper airway obstruction in the Shy-Drager syndrome. Ann Neurol 1977;2:83.

2 Williams A, Hanson D, Calne DB: Vocal cord paralysis in the Shy-Drager syndrome. J Neurol Neurosurg Psychiatry 1979;42:151-153.

3 Bannister R, Gibson W, Michaels L, Oppenheimer DR: Laryngeal abductor paralysis in multiple system atrophy. A report on three necropsied cases, with observations on the laryngeal muscles and the nuclei ambigui. Brain 1981;104:351-368.

4 Lapresle J, Annabi A: Olivopontocerebellar atrophy with velopharyngolaryngeal paralysis: a contribution to the somatotopy of the nucleus ambiguus. J Neuropathol Exp Neurol 1979;38:401-406.

5 Isozaki E, Shimizu T, Takamoto K, Horiguchi S, Hayashida T, Oda M, Tanabe H: Vocal cord abductor paralysis (VCAP) in Parkinson's disease: difference from VCAP in multiple system atrophy. J Neurol Sci 1995;130:197-202.

6 Hayashi M, Isozaki E, Oda M, Tanabe H, Kimura J: Loss of large myelinated nerve fibres of the recurrent laryngeal nerve in patients with multiple system atrophy and vocal cord palsy. J Neurol Neurosurg Psychiatry 1997;62:234-238.

7 Yoshihara T, Yamamura Y, Kaneko F, Abo N, Nomoto M: Neuromuscular junctions of the posterior cricoarytenoid muscle in multiple system atrophy: a case study. Acta Otolaryngol Suppl 2009;562:115-119.

8 Plasse HM, Lieberman AN: Bilateral vocal cord paralysis in Parkinson's disease. Arch Otolaryngol 1981;107:252-253.

9 Kim HJ, Jeon BS: Acute respiratory failure due to vocal cord paralysis in a patient with Parkinson's disease. Mov Disord 2009;24: 1862-1863.

10 McKeith IG, Dickson DW, Lowe J, et al; Consortium on DLB: Diagnosis and management of dementia with Lewy bodies: third report of the DLB consortium. Neurology 2005; 65: 1863-1872. 